\title{
Insulin and Heparin Therapies in Acute Pancreatitis due to Hypertriglyceridemia
}

\author{
Engin Altinkaya ${ }^{1}$ and Ahmet Aktas $^{2}$ \\ ${ }^{1}$ Department of Gastroenterology, Sivas Cumhuriyet University, Sivas, Turkey \\ ${ }^{2}$ Department of Internal Medicine, Sivas Cumhuriyet University, Sivas, Turkey
}

\begin{abstract}
Objective: To compare insulin infusion and heparin infusion, used to treat hypertriglyceridemia-induced acute pancreatitis in terms of efficacy.

Study Design: Descriptive study.

Place and Duration of Study: Department of Gastroenterology, Division of Internal Disease, Sivas Cumhuriyet University Hospital, Sivas, Turkey, from $1^{\text {st }}$ January 2013 to $1^{\text {st }}$ January 2020.

Methodology: Patients who presented with abdominal pain and were found to have edematous pancreatitis on computed tomography imaging were retrospectively reviewed. Thirty-six patients with hypertriglyceridemia were included. Eighteen of the patients were given insulin infusion, while the other 18 were given heparin infusion therapy. The patients' gender, age, admission amylase values, AST, ALT, total cholesterol, glucose, lactate dehydrogenase (LDH), CRP, leukocyte, pre-treatment and post-treatment first, second, and third-day triglyceride levels were recorded from the files.

Results: The post-treatment third-day triglyceride levels of the insulin therapy group were statistically significantly lower compared to the heparin therapy group $(432.5 ; 984, p<0.001$, respectively).

Conclusion: Insulin infusion, in addition to fluid therapy and electrolyte imbalance, was more effective than heparin therapy in patients with hypertriglyceridemia-induced acute pancreatitis.
\end{abstract}

Key Words: Hypertriglyceridemia-induced acute pancreatitis, Insulin therapy, Heparin therapy.

How to cite this article: Altinkaya E, Aktas A. Insulin and Heparin Therapies in Acute Pancreatitis due to Hypertriglyceridemia. J Coll Physicians Surg Pak 2021; 31(11):1337-1340.

\section{INTRODUCTION}

Acute pancreatitis is the most common gastrointestinal disease requiring hospital admission. In developed countries, its incidence is 34 per 100,000 people. ${ }^{1}$ Acute pancreatitis can usually present with local and systemic inflammatory response in variable clinical settings. Patients usually present with mild pancreatitis and theirclinic usually improve within one week. In approximately $20 \%$ of cases, acute pancreatitis is moderate or severe, and patients develop necrosis of the pancreatic tissue or organ failure. The mortality rate varies between 20 and $40 \%$ in cases of severe pancreatitis. ${ }^{2}$

Patients with acute pancreatitis usually present with severe upper abdominal pain. The diagnosis of acute pancreatitis is based on the presence of at least two of the three diagnostic criteria.

Correspondence to: Dr. Ahmet Aktas, Department of Internal Medicine, Sivas Cumhuriyet University, Sivas, Turkey

E-mail: ahmetaktas0142@hotmail.com

Received: January 17, 2021; Revised: March 18, 2021;

Accepted: March 30, 2021

DOI: https://doi.org/10.29271/jcpsp.2021.11.1337
The first is characteristic upper abdominal pain, the second is elevated serum amylase or lipase (or both) at least greater than three times the upper limit of normal, and the third is the presence of findings consistent with acute pancreatitis on computed tomography, magnetic resonance imaging, or ultrasonographic imaging. ${ }^{3}$ If typical clinical and laboratory findings are present, no additional imaging is required to confirm the diagnosis of acute pancreatitis. However, imaging can be performed at an early stage when diagnostic uncertainty is present. Necrotising pancreatitis can usually be detected on imaging 72 to 96 hours after the onset of symptoms. ${ }^{4}$

Gallstones (45\%) and alcohol use (20\%) are the most common causes of acute pancreatitis in developed countries. Other causes include medical therapy, endoscopic retrograde cholangiopancreatography (ERCP), hypercalcemia, hypertriglyceridemia, infections, genetic, autoimmune diseases, and surgical trauma. In acute pancreatitis cases, conditions such as alcohol consumption, drug use, family history, and gallstone history should be strictly reviewed. If the history, physical examination, and imaging reveal no factor for acute pancreatitis, more comprehensive imaging and laboratory tests should be carried out to prevent recurrence of pancreatitis. ${ }^{5,6}$

Hypertriglyceridemia is often an overlooked important cause of non-gallstone acute pancreatitis. It is thought to be account for 
approximately 1-4\% of all acute pancreatitis cases. Hypertriglyceridemia-induced acute pancreatitis is more common in secondary hypertriglyceridemias due to alcoholism, obesity, and uncontrolled diabetes. ${ }^{7}$ A triglyceride level above $500 \mathrm{mg} / \mathrm{dL}$ indicates a risk of acute pancreatitis, but its incidence is much higher, especially at levels of $1,000 \mathrm{mg} / \mathrm{dL}$ and above. While mild and moderate triglyceride elevation is observed in approximately $30 \%$ of all acute pancreatitis cases, hypertriglyceridemic

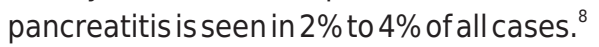

Treatment of hypertriglyceridemia-induced acute pancreatitis consists of aggressive fluid therapy, analgesia, correction of electrolyte imbalance, and triglyceride-lowering therapies. Insulin, heparin, anti-hypertriglyceridemic drugs, and therapeutic plasmapheresis can be used to lower the serum triglyceride level. $^{9}$

In this study, the aim was to compare the effectiveness of insulin and heparin therapies in lowering triglyceride levels of patients with hypertriglyceridemia-induced acute pancreatitis.

\section{METHODOLOGY}

Patients who presented to Faculty of Medicine, Cumhuriyet University Hospital between 2013-2020 with abdominal pain and who were found to have edematous pancreatitis on computed tomography imaging were retrospectively reviewed. Thirty-six patients with hypertriglyceridemia were included in this study. Eighteen of the patients were given insulin infusion, while the other 18 were given heparin infusion therapy. Insulin infusion was administered by adding $0.1-0.3 / \mathrm{Kg} / \mathrm{hour}$ crystalline insulin into $5 \%$ dextrose. Insulin infusion was titrated to maintain blood glucose above 200 by hourly monitoring of blood glucose. Heparin therapy was administered intravenously as 5000 units twice daily by maintaining APTT value below 100. The patients' gender, age, admission amylase values, AST, ALT, total cholesterol, glucose, lactate dehydrogenase (LDH), CRP, leukocyte, pre-treatment and post-treatment first, second, and third-day triglyceride levels were recorded from the files.

Statistical analysis of the data to be obtained was carried out using the SPSS 22.0 statistical software package (SPSS Inc., Chicago, IL, USA). The Shapiro-Wilk test was used for normally distributed parameters. The student's t-test was used to compare normally distributed parameters, and the MannWhitney U-test was used to compare non-normally distributed parameters. The exact statistics were noted as mean \pm standard deviation (SD) and median (interquartile, IQR) for quantitative data while frequency and percentage for qualitative data. The level of significance was set at 0.05 .

\section{RESULTS}

There were 36 patients, 11 females (30.6\%) and 25 males (69.4\%). Eighteen patients received insulin therapy, while the other 18 patients received heparin therapy. There was no significant difference between the insulin and heparin groups, age, calcium, WBC, total cholesterol, amylase, AST, CRP, LDH and triglyceride at admission. The ALT value was found below to be significant in the group receiving insulin treatment compared to the group receiving heparin therapy. Although there was no statistically significant difference between the triglyceride levels on the first day and the second day of treatment in both groups, it was found to be statistically significantly lower in the group receiving insulin treatment on the third day of the treatment compared to the group receiving heparin treatment (Table I).

Table I: Comparison of demographic and laboratory values according to the treatmentgroup.

\begin{tabular}{|c|c|c|c|}
\hline & Insulın & Heparın & \multirow[b]{2}{*}{$\mathbf{p}$} \\
\hline & $\begin{array}{c}\text { Mean } \pm \text { S.D/ } \\
\text { Median (IQR) }\end{array}$ & $\begin{array}{c}\text { Mean } \pm \text { S.D/ } \\
\text { Median (IQR) }\end{array}$ & \\
\hline Age (year) & $49.94 \pm 12.04$ & $51.55 \pm 11.60$ & 0.685 \\
\hline Calcium (mg/dL) & $9.18 \pm 0.77$ & $9.34 \pm 0.54$ & 0.491 \\
\hline WBC & $11166 \pm 3581.30$ & $8933.89 \pm 3010.34$ & 0.051 \\
\hline Total cholesterol & $347.88 \pm 122.43$ & $397.44 \pm 156.96$ & 0.298 \\
\hline Amylase & $\begin{array}{c}1861.5 \\
(1422.5-2022)\end{array}$ & $\begin{array}{c}1807.5 \\
(1599.5-2100)\end{array}$ & 0.924 \\
\hline AST & $\begin{array}{c}43 \\
(25.25-92.25)\end{array}$ & $\begin{array}{c}27 \\
(22.75-47.25)\end{array}$ & 0.154 \\
\hline ALT & $\begin{array}{c}60 \\
(31.75-118.0) \\
\end{array}$ & $\begin{array}{c}28.5 \\
(19.5-48.25) \\
\end{array}$ & $0.015^{*}$ \\
\hline Glucose & $\begin{array}{c}173 \\
(135.5-190.5)\end{array}$ & $\begin{array}{c}207 \\
(165-313.25)\end{array}$ & $0.041^{*}$ \\
\hline LDH & $\begin{array}{c}239 \\
(195.25-302)\end{array}$ & $\begin{array}{c}214 \\
(188.25-247)\end{array}$ & 0.229 \\
\hline CRP & $\begin{array}{c}7.5 \\
(3.28-13.75)\end{array}$ & $\begin{array}{c}5.685 \\
(3.28-12.8)\end{array}$ & $>0.999$ \\
\hline $\begin{array}{l}\text { Triglyceride } \\
\text { (mg/dL) }\end{array}$ & $\begin{array}{c}2533.5 \\
(2166.75-3361)\end{array}$ & $\begin{array}{c}2597.5 \\
(2186.75-3725)\end{array}$ & 0.849 \\
\hline
\end{tabular}

Table II: Comparison of laboratory values of insulin group and heparin group before and after treatment.

\begin{tabular}{|l|c|c|c|}
\hline & Insulın & Heparın & \multirow{2}{*}{$\mathbf{p}$} \\
\cline { 2 - 3 } & Median (IQR) & Median (IQR) & \\
\hline $\begin{array}{l}\text { Post-treatment first-day } \\
\text { Triglyceride (mg/dL) }\end{array}$ & $\begin{array}{c}1636 \\
(1077.75-2015.25)\end{array}$ & $\begin{array}{c}1305 \\
(1135.25-1873.75)\end{array}$ & 0.658 \\
\hline $\begin{array}{l}\text { Post-treatment second- } \\
\text { day Triglyceride (mg/dL) }\end{array}$ & $\begin{array}{c}1083 \\
(706.5-1276.75)\end{array}$ & $\begin{array}{c}1125.5 \\
(897.75-1319)\end{array}$ & 0.393 \\
\hline $\begin{array}{l}\text { Post-treatment third-day } \\
\text { Triglyceride (mg/dL) }\end{array}$ & $\begin{array}{c}432.5 \\
(320.75-506)\end{array}$ & $\begin{array}{c}984 \\
(628-1125)\end{array}$ & $<0.001$ \\
\hline
\end{tabular}

There was no statistically significant difference between the insulin therapy and heparin therapy groups in terms of age, amylase level, admission, post-treatment first-day, post-treatment second-day ( $p>0.05)$. The post-treatment third-day triglyceride levels of the insulin therapy group were statistically significantly lower compared to the heparin therapy group ( $p$ $<0.05$, Table II).

\section{DISCUSSION}

Hypertriglyceridemia-induced acute pancreatitis is often overlooked since it is relatively less common than other causes of pancreatitis. ${ }^{10}$ The pancreas contains a high concentration of lipase. Pancreatic lipase hydrolyses triglycerides to glycerol and free fatty acids. In normal conditions, serum free fatty acids bind to albumin and are non-toxic. If high serum triglycerides are hydrolysed in the pancreas or if high serum triglycerides are hydrolysed in the pancreas by endogenous lipase, locally high 
concentrations of free fatty acids may develop, causing vascular endothelial damage, stasis, and ischemic injury to the pancreas with the release of large amounts of cytotoxic free fatty acids in the pancreatic circulation. ${ }^{11}$

In the treatment of hypertriglyceridemia-induced acutepancreatitis, insulin infusion, heparin therapy, triglyceride-lowering agents, and plasmapheresis can be used to reduce elevated triglyceride levels in addition to fluid therapy, analgesic, and regulation of electrolyte imbalance. ${ }^{12}$ Insulin therapy reduces triglyceride levels by inhibiting hormone-sensitive lipase and increasing lipoprotein lipase activity. ${ }^{13}$ Heparin therapy also increases lipoprotein lipase activity, decreases triglyceride levels, and acts by preventing thrombi that develops due to systemic inflammation. ${ }^{14}$ Alagozlu et al. compared insulin infusion and heparin therapy in patients with hypertriglyceridemia-induced acute pancreatitis and found insulin infusion safer in patients with diabetes. In addition to insulin and heparin, they also initiated their patients on triglyceride-lowering agents such as fenofibrate. ${ }^{15}$ Hammond et al. administered insulin infusion and heparin therapy in combination to five patients with hypertriglyceridemia-induced acute pancreatitis and found their triglyceride-lowering effects both effective and reliable. ${ }^{16}$ In a study including 106 patients with hypertriglyceridemia-induced acute pancreatitis, Dhindsa et al. compared insulin infusion and fluid therapy and found that the triglyceride-lowering effect of insulin infusion was not different from fluid therapy. ${ }^{17}$ In a study of 100 patients with hypertriglyceridemia-induced acute pancreatitis, Tozlu et al. found that heparin therapy did not make a difference in mortality versus standard fluid therapy, but that local complications were statistically significantly less common in patients receiving subcutaneous heparin therapy. ${ }^{18}$

In this study, 36 patients with hypertriglyceridemia-induced acute pancreatitis, were analysed; half of them received insulin therapy and the other half received heparin therapy. There was no significant difference between the patients in both treatment groups in terms of age, admission amylase levels, triglyceride levels, leukocyte count, CRP levels, and LDH levels. When we compared the patients receiving insulin therapy and heparin therapy, the post-treatment third-day triglyceride levels of the insulin therapy group were found to be statistically significantly lower compared to the heparin therapy group $(p<0.05)$.

The previous studies have compared heparin and insulin therapy withstandard fluid therapy in patients with hypertriglyceridemia-induced acute pancreatitis. In this study, authors compared heparin therapy and insulin therapy in hypertriglyceridemia-induced acute pancreatitis. Although there was no difference between the two groups in terms of complications, the authors found that the triglyceride-lowering effect of insulin therapy was more significant than that of heparin therapy. The small sample size of this study constitutes the limitation of this study. There is a need for comprehensive studies to more effectively demonstrate the triglyceride-lowering effect of insulin therapy.

\section{CONCLUSION}

Insulin infusion, in addition to fluid therapy and electrolyte imbalance was more effective than heparin therapy in patients with hypertriglyceridemia-induced acute pancreatitis.

\section{ETHICALAPPROVAL:}

Permission was taken from Cumhuriyet University Ethics Committee vide No. 2019-12/02, dated 11.12.2019.

\section{CONFLICT OF INTEREST:}

The authors declared no conflict of interest.

\section{AUTHORS' CONTRIBUTION:}

EA: Set up the main idea and hypothesis of the study.

AA, EA: Developed the theory and edited the methodology section.

AA: Made the evaluation of the data in the results section.

The discussion part of the article was written by AA and EA, AA reviewed and made the necessary corrections and approved. In addition, all authors discussed the entire study and approved its final version.

\section{REFERENCES}

1. Bálint ER, Für G, Kiss L, Németh DI, Soós A, Hegyi $P$, et al. Assessment of the course of acute pancreatitis in the light of aetiology: A systematic review and meta-analysis. Sci Rep 2020; 10(1):1-17. doi: 10.1038/s41598-020-74943-8.

2. Van den Berg FF, de Bruijn AC, van Santvoort HC, Issa Y, Boermeester MA. Early laboratory biomarkers for severity in acute pancreatitis: A systematic review and metaanalysis. Pancreatol 2020; 20(7):1302-11. doi: 10.1016/ j.pan.2020.09.007.

3. Mashayekhi R, Parekh VS, Faghih M, Singh VK, Jacobs MA, Zaheer A. Radiomic features of the pancreas on CT imaging accurately differentiate functional abdominal pain, recurrent acute pancreatitis, and chronic pancreatitis. Eur J Radiol 2020; 123:108778. doi: 10.1016/j.ejrad.2019. 108778.

4. Li Y, Zhang J, Zou J. Evaluation of four scoring systems in prognostication of acute pancreatitis for elderly patients. BMC Gastroenterol 2020; 20(1):1-7. doi: 10.1186/ s12876-020-01318-8.

5. Pu W, Luo G, Chen T, Jing L, Hu Q, Li X, et al. A 5-year retrospective cohort study: epidemiology, etiology, severity, and outcomes of acute pancreatitis. Pancreas 2020; 49(9):1161-7. doi: 10.1097/MPA.0000000 000001637.

6. Matta B, Gougol A, Gao X, Reddy N, Talukdar R, Kochhar R, et al. Worldwide variations in demographics, management, and outcomes of acute pancreatitis. Clin Gastroenterol Hepatol 2020; 18(7):1567-75. e2. doi: 10.1016/j.cgh. 2019.11.017.

7. Zádori N, Gede N, Antal J, Szentesi A, Alizadeh H, Vincze Á, et al. Early elimination of fatty acids iN hypertriglyceridemia-induced acuTe pancreatitis (ELEFANT trial): Protocol of an open-label, multicenter, adaptive randomised clinical trial. Pancreatol 2020; 20(3):369-76. doi: 10.1016/j.pan.2019.12.018. 
8. De Pretis N, De Marchi G, Frulloni L. Hypertriglyceridemic pancreatitis. Minerva Gastroenterol e Dietol 2020; 66(3):238-45. doi: 10.23736/S1121-421X.19.02641-2.

9. Wolska A, Yang ZH, Remaley AT. Hypertriglyceridemia: New approaches in management and treatment. Curr Opin Lipidol 2020; 31(6):331-9. doi: 10.1097/MOL.0000000 000000710.

10. Berberich A, Ziada A, Zou G, Hegele R. Conservative management in hypertriglyceridemia-associated pancreatitis. J Int Med 2019; 286(6):644-50. doi: 10. 1111/joim.12925.

11. McKitish K, Lee C, Sotiriadis J. Complications from hypertriglyceridemia-induced acute pancreatitis: 3029. Am J Gastroenterol 2019; 114(2019 ACG Annual Meeting Abstracts):S1636.

12. Parhofer KG, Laufs U. The diagnosis and treatment of hypertriglyceridemia. Deutsches Ärzteblatt Intern 2019; 116(49):825. doi: 10.3238/arztebl.2019.0825.

13. Soliman S. Hypertriglyceridemia-induced pancreatitis with rapid response to insulin therapy. J Med Cases 2020; 12(1):23-6. doi.org/10.14740/jmc3595.
14. Camargo-Mendoza JP, Rodríguez-Ariza DE, Bustos-Calvo JS. Heparin and insulin in the management of acute hypertriglyceridemic pancreatitis. Acta Medica Colombiana 2020; 45(1):44-7.

15. Alagözlü $H$, Cindoruk $M$, Karakan $T$, Ünal S. Heparin and insulin in the treatment of hypertriglyceridemia-induced severe acute pancreatitis. Digestive Dis Sci 2006; 51(5):931-3. doi: 10.1007/s10620-005-9006-z.

16. Hammond DA, Finlay L. Treatment of hypertriglyceridemiainduced acute pancreatitis with insulin, heparin, and Gemfibrozil: A case series. Hospital Pharm 2017; 52(10): 675-8. doi: 10.1177/0018578717725168.

17. Dhindsa S, Sharma A, Al-Khazaali A, Sitaula S, Nadella S, McKee $A$, et al. Intravenous insulin versus conservative management in hypertriglyceridemia-associated acute pancreatitis. J Endocrine Soc 2020; 4(1):bvz019. doi: 10.1210/jendso/bvz019.

18. Tozlu M, Kayar Y, Ince AT, Baysal B, Şentürk H. Low molecular weight heparin treatment of acute moderate and severe pancreatitis: A randomised, controlled, open-label study. Turk J Gastroenterol 2019; 30(1):81-7. doi: 10.5152/tjg.2018.18583. 